\title{
Tuberculosis (TB) in the hispanic population: an ongoing problem
}

\section{Opinion}

My capstone project for this course is to address the increased numbers of reported tuberculosis cases among the Hispanic population and discuss ways that can help reduce the number of incidences. ${ }^{1}$ This project is to help examine my strengths I've gained from my experience at Walden to advocate social change.

Tuberculosis is a bacteria that affects the lungs, spine, kidneys and brain. It can lead to death if not treated correctly and quickly and it is caused by the bacteria Mycobacterium tuberculosis. ${ }^{2}$ It is airborne which means it is spread through the air from one person to another. When a person with active tuberculosis coughs, sneezes, laughs, anyone nearby can breathe in the bacteria and becomes infected but a person cannot get infected by kissing, touching or shaking hands, sharing food or toilet seats. ${ }^{2}$ There are two types of tuberculosis; latent tuberculosis infection and tuberculosis disease. With latent tuberculosis infection the bacteria is in the body without the person becoming sick and cannot spread the bacteria to others. ${ }^{2}$ The person with active tuberculosis becomes sick and passes it to another person. The symptoms of tuberculosis disease are pain in the chest, loss of appetite, night sweats, chills, weight loss, and cough lasting 3 weeks or more. ${ }^{2}$

According to the CDC in 2012, nationally there were 9,945 tuberculosis cases reported. From that report $29 \%$ were from the Hispanic population making them the largest percentage of totaled reported tuberculosis cases in the United States after Asians. According to Cardenas \& Kerby, ${ }^{3}$ the 2010 U.S. Census Bureau Hispanic population is 50.5 million that is $16 \%$ of the U.S. population. By 2050 the Hispanic population should double to $30 \%$. They are the fastest-growing population in the U.S. and continue to face disparities, such as education, employment, health and political leadership. Employed Hispanics are less likely to have a college degree and are grouped in certain industries like farm work. ${ }^{3}$ They are more likely to move within a county for employment opportunities. Hispanic adults who are not citizens or have legal residence lack health insurance and health care access. Cardenas \& Kerby ${ }^{3}$ noted that the quality of health care is lower than those of other racial group; they experience worse health outcomes and higher rate of certain illness. The illness this population face are childhood obesity, women are likely to die from pregnancy complication twice the amount of whites, Hispanic women have a higher percentage to die from breast cancer than that of white women. Hispanics are three times more likely than whites to be infected with HIV/AIDS, and they exceed all racial groups with the largest reported tuberculosis cases in the United States.

The risk factors for developing tuberculosis is higher if a person has HIV, health problems like diabetes, use illegal drugs, not treated properly from a previous tuberculosis infection. ${ }^{2}$ There are more reported cases of TB-HIV co-infection trends in San Diego, CA Hispanic population. There were $82 \%$ reported cases of TB-HIV co-infection in 2007. ${ }^{4}$ San Diego's Hispanic population is over 30\% and most of those populations were born in Mexico. Most of the people in this population may have entered the United States with
Volume 3 Issue 2 - 2015

\author{
Tanicka Shelton \\ Walden University, Department of Health Services, USA
}

Correspondence: Tanicka Shelton Walden University,

Department of Health Services, USA

Email tanickash@yahoo.com

Received: December 30, 2014 | Published: December 15, 2015

latent tuberculosis or active tuberculosis and did not know it. Of that population, $10 \%$ of foreign born TB/HIV co-infected reported living in the United States less than 90 days which makes it likely that they were infected with HIV in Mexico and not the United States. ${ }^{4}$ Migrant workers are at greater risk for HIV infection when they first enter the United States because of individual behaviors. Injected drug use was associated with TB-HIV co-infection and it suggested that these patients be administered HIV risk assessment surveys when doing TB surveillance in San Diego in order to help develop an understanding of the risk profile of the individuals. Because of the close proximity to Mexico and Tijuana the infection rate in San Diego may be different than California as a whole. ${ }^{5}$

Ailinger et al., ${ }^{6}$ noted that people who has latent tuberculosis are asymptomatic meaning they have no symptoms of the disease, about $5-10 \%$ will become sick. ${ }^{6}$ They stated treating patients with latent tuberculosis can help with the elimination of tuberculosis in the United States. Latent tuberculosis treatment is done to prevent the progression of active tuberculosis in the United States. People that come from other countries where tuberculosis is endemic have an increased chance of being infected with tuberculosis. Hispanics account for $29 \%$ of active tuberculosis cases which is high for their population. ${ }^{2}$ There would be fewer active tuberculosis cases in the Hispanic population if they would continue with their INH regimen. There were several factors that influenced adherence to latent tuberculosis treatment in Hispanic population; these factors are access to healthcare facilities, length of time to complete treatment and the confusion of the healthcare system, and the side effects of medication. In a study of 286 Hispanic teens that were put in different groups the group that showed the highest completed treatment rate were those that received medical care and coaching. ${ }^{6}$

Cardenas \& $\mathrm{Kerby}^{3}$ noted some environmental factors that can affect treatment adherence for tuberculosis and they are emotional beliefs, daily work schedule, seasonal work, and transportation. Lack of knowledge, transmission and treatment of latent tuberculosis, stigma of tuberculosis also are factors with non-compliance of treatment. To help adhere to treatment one should address the sociocultural factors of the Hispanic population. Sociocultural factors are language barriers, adjusting clinic hours and supplying transportation for clinic appointments. 
Incorporating cultural intervention along with nursing care for Hispanic immigrant patients with latent tuberculosis show some improvements. Cultural intervention can be cost effective and should be encouraged in research that can be used to build evidence for culturally appropriate practice.

$\mathrm{CDC}$ fact sheet ${ }^{5}$ states that multidrug resistant tuberculosis (MDR) is tuberculosis that are resistant to isoniazid and rifampin the 2 most common and effective medication to treat tuberculosis. Multidrug resistant tuberculosis is prevalent in Hispanics born outside of the United States. Tuberculosis control globally is decreasing tuberculosis incidence but the emergence of multidrug resistant tuberculosis can make tuberculosis an untreatable disease. California had an average of 41 MDR from 1994-2003 which is the highest in the United States. $85 \%$ of MDR incidence in California were foreign born and $28 \%$ of this amount were from Mexico ${ }^{2}$ California and Baja California share a high migratory rate. California law says that all tuberculosis cases be submitted to local public health labs to be tested for drug susceptibility. Patients that were recently diagnosed with tuberculosis were classified as Mexicans living in Baja California, Mexico born Hispanics living in California and US born Hispanics living in California. The testing population showed that there might be a similar tuberculosis strain between California and Baja California and that the patients living in Mexico are more likely susceptible to have MDR-TB because of the resources not available to them. Individuals that share the same sociocultural characteristics and social networks through which TB can be transmitted have different prevalence of multidrug resistant tuberculosis. For multidrug resistant tuberculosis to be controlled governments and society need to find ways to face challenges of financing, program organization, laboratory capacity, and political commitment, if not addressed it will likely worsen over time. $^{2}$

With the Hispanic population increasing and becoming the largest minority group in the United States, the prevalence of contracting tuberculosis is greater. Without taking preventive measures to reduce the rate of reported tuberculosis cases it will continue to be an ongoing problem in the United States. Understanding the Hispanic culture, continued research and education, and increased funding toward preventive measures in this population, the high amount of incidences will decrease over time.

\section{Acknowledgements}

None.

\section{Conflict of interest}

The author declares no conflict of interest.

\section{References}

1. Centers for Disease Control and Prevention (CDC). Health Disparities in HIV/AIDS, Viral Help, STD and TB.

2. Bojorquez I, Barnes RF, Flood J, et al. Multidrug-resistant Tuberculosis among patients in Baja California, Mexico, and Hispanic patients in California. American Journal of Public Health. 2013;103(7):13011305.

3. Cardenas V, Kerby S. The State of Latinos in the United States. Center for American Progress; 2012.

4. Rodwell TC, Barnes RFW, Moore M, et al. HIV-Tuberculosis Coinfection in Southern California: Evaluating Disparities in Disease Burden. American Journal of Public Health. 2010;100(Suppl 1):S178S185.

5. CDC. Tuberculosis (TB) Fact Sheet: Tuberculosis in Hispanics/Latinos. Centers for Disease Control and Prevention; 2013.

6. Ailinger RL, Martyn D, Lasus $\mathrm{H}$, et al. The effect of a cultural intervention on adherence to latent tuberculosis infection therapy in Latino Immigrants. Public Health Nursing. 2010;27(2):115-120. 